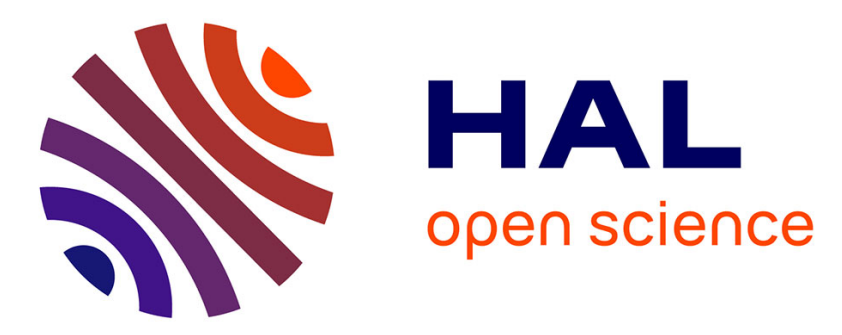

\title{
Evaluation of deep moonquake source parameters: Implication for fault characteristics and thermal state
}

Taichi Kawamura, Philippe Lognonné, Yasuhiro Nishikawa, Satoshi Tanaka

\section{To cite this version:}

Taichi Kawamura, Philippe Lognonné, Yasuhiro Nishikawa, Satoshi Tanaka. Evaluation of deep moonquake source parameters: Implication for fault characteristics and thermal state. Journal of Geophysical Research. Planets, 2017, 122 (7), pp.1487-1504. 10.1002/2016JE005147 . insu-02553247

\section{HAL Id: insu-02553247 \\ https://hal-insu.archives-ouvertes.fr/insu-02553247}

Submitted on 6 Aug 2020

HAL is a multi-disciplinary open access archive for the deposit and dissemination of scientific research documents, whether they are published or not. The documents may come from teaching and research institutions in France or abroad, or from public or private research centers.
L'archive ouverte pluridisciplinaire HAL, est destinée au dépôt et à la diffusion de documents scientifiques de niveau recherche, publiés ou non, émanant des établissements d'enseignement et de recherche français ou étrangers, des laboratoires publics ou privés. 


\section{Journal of Geophysical Research: Planets}

\section{RESEARCH ARTICLE \\ 10.1002/2016JE005147 \\ Key Points: \\ Evaluation of deep moonquake source parameters: Implication for fault characteristics and thermal state}

- Reevaluated source parameters of deep moonquakes through spectral analyses with combined spectra from long-and short-period seismic records of Apollo

- Deep moonquake faults are better explained with a self-affine model than a self-similar model and likely to be smoother than terrestrial faults

- Brittle-ductile transition temperature was reevaluated and was compared to temperature profiles of the Moon

Correspondence to:

T. Kawamura,

kawamura@ipgp.fr;

t.kawamura@nao.ac.jp

\section{Citation:}

Kawamura, T., P. Lognonné,

Y. Nishikawa, and S. Tanaka (2017),

Evaluation of deep moonquake source

parameters: Implication for fault

characteristics and thermal state,

J. Geophys. Res. Planets, 122, 1487-1504, doi:10.1002/2016JE005147.

Received 1 AUG 2016

Accepted 16 JUN 2017

Accepted article online 22 JUN 2017

Published online 18 JUL 2017

(c)2017. American Geophysical Union. All Rights Reserved.

\author{
Taichi Kawamura ${ }^{1,2}$, Philippe Lognonné ${ }^{1}$, Yasuhiro Nishikawa ${ }^{1,3}$, and Satoshi Tanaka ${ }^{4}$ \\ ${ }^{1}$ Institut de Physique du Globe de Paris, Université Paris Diderot-Sorbonne Paris Cité, Paris, France, ${ }^{2}$ National Astronomical \\ Observatory of Japan, Oshu City, Japan, ${ }^{3}$ Department of Earth and Planetary Science, The University of Tokyo, Bunkyo, \\ Japan, ${ }^{4}$ Institute of Space and Astronautical Science, Japan Aerospace Exploration Agency, Sagamihara, Japan
}

Abstract While deep moonquakes are seismic events commonly observed on the Moon, their source mechanism is still unexplained. The two main issues are poorly constrained source parameters and incompatibilities between the thermal profiles suggested by many studies and the apparent need for brittle properties at these depths. In this study, we reinvestigated the deep moonquake data to reestimate its source parameters and uncover the characteristics of deep moonquake faults that differ from those on Earth. We first improve the estimation of source parameters through spectral analysis using "new" broadband seismic records made by combining those of the Apollo long- and short-period seismometers. We use the broader frequency band of the combined spectra to estimate corner frequencies and DC values of spectra, which are important parameters to constrain the source parameters. We further use the spectral features to estimate seismic moments and stress drops for more than 100 deep moonquake events from three different source regions. This study revealed that deep moonquake faults are extremely smooth compared to terrestrial faults. Second, we reevaluate the brittle-ductile transition temperature that is consistent with the obtained source parameters. We show that the source parameters imply that the tidal stress is the main source of the stress glut causing deep moonquakes and the large strain rate from tides makes the brittle-ductile transition temperature higher. Higher transition temperatures open a new possibility to construct a thermal model that is consistent with deep moonquake occurrence and pressure condition and thereby improve our understandings of the deep moonquake source mechanism.

Plain Language Summary Apollo seismic observation discovered that the Moon is seismically active and the observation detected more than 13000 seismic events. Among the detected events, most frequently observed seismic events are deep moonquakes that occur at 800-1200 km depth in the Moon. Although intensive studies have been carried out for decades, why and how deep moonquakes occur are remaining mysteries. One of the reasons is that the magnitude of the deep moonquake fault and the speed of the seismic slip are unclear. In this study, we reinvestigated the deep moonquake data to reveal their fault characteristics. While previous studies mainly used the long-period seismometer records, we also used the short-period seismic records of Apollo to improve the estimation. This enabled us to study more than 100 deep moonquakes events while only one event was studied in the previous study. With 131 deep moonquakes, we carried out comparative and statistic study of the fault characteristics. Our study revealed that the deep moonquake faults are much smoother compared to the terrestrial counterparts and the stress release of the event is as low as the tidal stress between the Earth and the Moon. The correlation between the deep moonquake occurrence and tidal stress has been pointed out and our study supports the idea that the tidal stress is not only triggering the deep moonquakes but also responsible of the whole stress release of the seismic activity.

\section{Introduction}

Deep moonquakes are the most frequently observed seismic events on the Moon, and more than 7000 events were detected during the Apollo seismic experiment (July 1969 to September 1977) [Nakamura, 2003] (Figure 1). In spite of the many recorded deep moonquake events, the exact nature of deep moonquake occurrence remains to be understood. Understanding the source mechanisms of deep moonquakes will open a window on the mechanical and thermal conditions of the deep interior of the Moon and will provide a unique opportunity to observe active features of the deep interior of the Moon. Deep moonquake source 


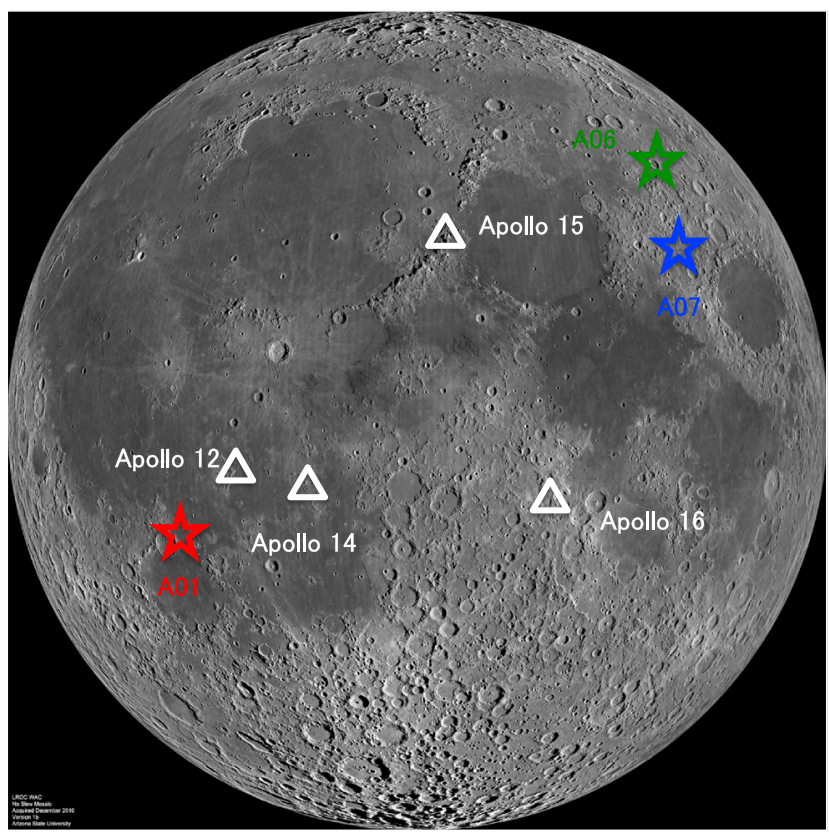

Figure 1. Geometric configuration of Apollo seismic stations and deep moonquake source regions. The lunar globe was taken from Lunar Reconnaissance Orbiter Camera (LROC) observation courtesy of NASA (http://photojournal.jpl.nasa.gov/catalog/PIA14011). Apollo stations and deep moonquake nests were added by the authors. regions are located between depths of 750 and $1200 \mathrm{~km}$ in the most recent analyses [Khan and Mosegaard, 2002; Lognonné et al., 2003; Nakamura, 2003]. They occur periodically with the tides raised on the Moon by the Earth and the Sun at well-defined nests, suggesting their triggering by the terrestrial tide [e.g., Lammlein, 1977].

Various studies have evaluated the tidal stress acting on deep moonquake source regions [Toksöz et al., 1977; Cheng and Toksöz, 1978; Minshull and Goulty, 1988; Weber et al., 2009]. While these studies succeeded in giving quantitative estimates of the tidal stress, they were not able to fully describe the source activity of deep moonquakes. Among the reasons for this are the poor constraints on the source parameters of deep moonquakes, such as seismic moment and stress drop that result from both the poor data quality of Apollo and the deep moonquakes' low magnitudes [Goins et al., 1981].

The aim of this study is to revisit the Apollo seismic data and carry out an improved estimation of seismic moments and stress drops of deep moonquakes, which will provide new constraints on the source mechanism including mechanical and thermal conditions at the depth of the deep moonquakes. Better understanding of the source mechanisms of deep moonquakes and comparison of the tidal strain/stress drop ratio with respect to the brittle-ductile transition are critical for understanding the lunar seismic activity.

\subsection{Existing Problems of the Deep Moonquake Source Mechanism}

Two major issues regarding the investigation of the deep moonquake source mechanism still exist. The first is the relation between stress drop, tidal stress, and lithostatic pressure. Only the study by Goins et al. [1981] quantitatively evaluated the source parameters of deep moonquakes and estimated the source parameters for the largest event from the A01 deep moonquake. They obtained a stress drop of approximately $0.01 \mathrm{MPa}$ and a seismic moment of $5 \times 10^{13} \mathrm{Nm}$. Although previous studies claim that the normal and shear tidal stress (0.1-0.01 MPa) [Toksöz et al., 1977; Cheng and Toksöz, 1978; Minshull and Goulty, 1988; Weber et al., 2009] are in good agreement with such a stress drop, how such a slip with a small stress drop is triggered under high lithostatic pressure ( 4 GPa) still remains unclear. Goins et al. [1981] considered only one deep moonquake event. Therefore, whether the values are representative of all deep moonquakes or they should be regarded as an outlier estimated with the largest event is unclear. To better understand the characteristics of deep moonquakes, providing estimates for a larger number of events and having a global and statistical view of the source parameters are important.

The second issue concerns the thermal conditions of the source region. Several studies have proposed thermal profiles of the Moon, suggesting that the temperature of the deep moonquake source region is too high for it to be in a brittle regime [e.g., Gagnepain-Beyneix et al., 2006; Khan et al., 2014]. The recent discovery of the liquid state of the lunar core [Weber et al., 2011; Garcia et al., 2011] and strong tidal heating and/or partial melting at the base of the mantle [Harada et al., 2014; Khan et al., 2014] also suggests high temperatures at the lunar core mantle boundary. At such temperatures (1500-1600 K) and pressures $(\sim 4 \mathrm{GPa})$, the source regions are likely to be in a ductile regime, and an ordinary brittle fracture is not likely to be possible. 
Seismic activity in ductile conditions is also observed on Earth [e.g., Ye et al., 2016; Frohlich and Nakamura, 2009]. Comparison between deep moonquakes and intermediate-depth earthquakes is discussed in detail in Frolich and Nakamura [2009]. In terms of pressure, deep moonquakes correspond to intermediatedepth earthquakes at a depth of 100-135 km. Both cases occur at the pressure and temperature conditions wherein ordinary brittle fracture is unlikely. On Earth, these intermediate-depth earthquakes are now recognized as those occurring in the cold, stressed, subducting lithosphere [Frolich and Nakamura, 2009]. Several models have been proposed for the possible source mechanisms for such intermediate-depth earthquakes. The first model is transformational faulting, which is related to the phase transition of minerals [Hacker et al., 2003]. However, this model is not suitable for the mechanism of deep moonquakes because based on the pressure and temperature conditions of the source regions, any phase transformation is not likely to occur [e.g., Khan et al., 2007; Kuskov and Kronrod, 2009]. The second is plastic or melt instabilities [Hacker et al., 2003]. This model is mainly used to explain deep earthquakes at $>300 \mathrm{~km}$ depth, where the pressure and temperature are higher than those of deep moonquake source regions. There is no clear evidence of its application to intermediate-depth earthquakes [Hacker et al., 2003]. Whether this model can explain the source mechanism of deep earthquakes is not evident. Thus, its application to deep moonquakes also needs to be carefully considered. The third is dehydration embrittlement, which is expected to occur when minerals release a fluid that increases the pore pressure [Hacker et al., 2003]. This is a widely accepted explanation for seismicity in a subduction zone, where water contents are 0.1-5 wt \%. Recent discovery of water on the Moon [e.g., Saal et al., 2008] questions whether the lunar interior is dry; however, the expected water content in the subduction zone is considerably lower than that in the terrestrial subduction zones wherein dehydration embrittlement plays a significant role. Thus, although several studies suggest possible explanations for intermediate-depth earthquakes, applying them to explain the seismicity of deep moonquakes is difficult.

\subsection{Outline of This Study}

In this study, we first perform spectral analysis of Apollo seismic data and use the obtained source parameters to reevaluate the thermal conditions of the source regions. The analysis method followed here is outlined in the scheme shown in Figure 2. First, spectral analyses of Apollo seismic data are performed for estimating the spectral features of deep moonquake spectra to constrain the source time function of deep moonquakes (section 2). Assuming that deep moonquakes are fault activities that can be expressed with double-coupled force, we estimate the stress drops of deep moonquakes from the source time functions (section 3). With the obtained stress, which is comparable to the tidal stress, we assume that the main source of stress glut, causing deep moonquakes, is tidal stress. Next, we estimate the brittle-ductile transition temperature for deep moonquakes using tidal strain rates (sections 4.1-4.3). Following the discussion in section 1.1 and with analogy to intermediate-depth earthquakes, we assume that deep moonquakes are triggered via brittle fractures in the source regions. To quantitatively compare the existing temperature models of the Moon and the brittleductile transition temperature, reevaluation of the transition temperature is essential. The obtained transition temperature will then be used to constrain the temperature profile inside the Moon (section 4.3). Assuming that deep moonquakes occur as a result of brittle fractures on faults, the temperature in the deep moonquake source regions is required to be lower than the transition temperature. We compared the obtained brittleductile temperature with several temperature profiles of the Moon proposed in previous studies to test whether the transition temperature is compatible with the existing models (section 4.4).

\section{Data Processing}

The rupture signature of deep moonquakes can be extracted from the source time function within seismic spectra. However, the limited frequency band and instrument sensitivity of the Apollo seismometers have prevented detailed spectral analysis. In addition to this, the spectral features are masked by the propagation and site reverberation effects, the latter being large on the Moon due to the high scattering and low attenuation. We overcome these difficulties by (1) combining Apollo long-period (LP) and short-period (SP) data to obtain the equivalent of broadband data, (2) stacking the signals for the same pair of stations and deep moonquake nests to evaluate the nonseismic source term within the spectra, and (3) applying a correction factor to account for the energy redistributed into the coda by the intense scattering [e.g., Goins et al., 1981]. 


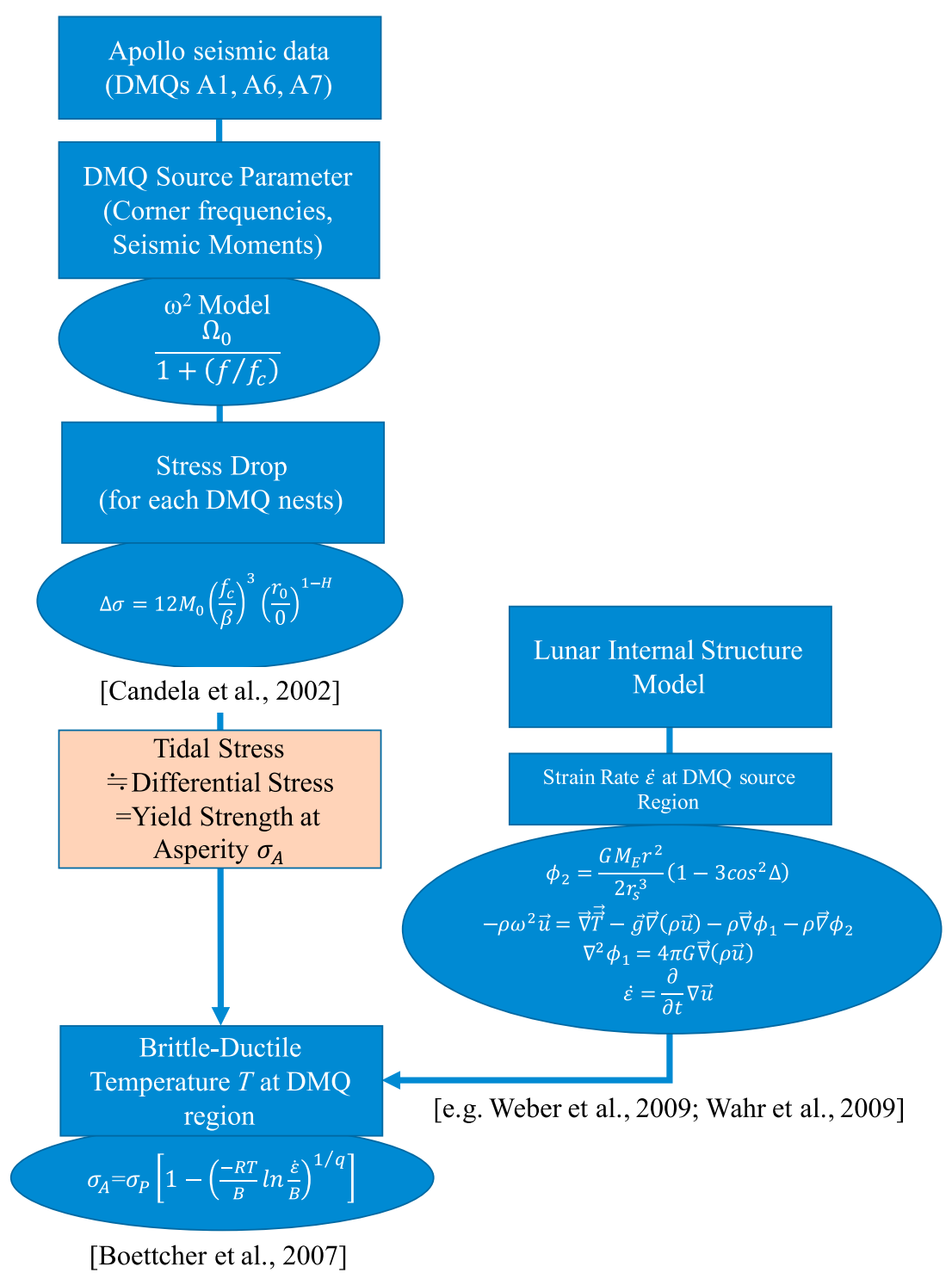

Figure 2. Flowchart of the data processing carried out in this study.

\subsection{Recombination of Long-Period (LP) and Short-Period (SP) Seismic Data}

While estimation of the source parameters through spectral analysis has already been performed by Goins et al. [1981], it suffered from the limited frequency band of the LP Apollo seismometer. The corner frequencies of seismic records are an important parameter that quantifies the source parameters such as slip time, seismic moment, and seismic energy release. Goins et al. [1981] evaluated the corner frequency of the largest deep moonquake with LP data and reported $1 \mathrm{~Hz}$ for the corner frequency. However, this value is close to the upper limit of the LP frequency band and may contain large uncertainties, especially when the true corner frequency is higher (Figure 3). Each Apollo Lunar Surface Experiment Package also contained one vertical SP seismometer, and the Apollo LP and SP seismometers cover different frequency bands with some overlap (Figure 3; LP: $0.1-1.5 \mathrm{~Hz}$ and SP: 1-10 Hz). Since stress drops of seismic activities are proportional to the corner frequency with a power of 3 , a small difference in the corner frequencies can result in a large difference in the stress drops [e.g., Aki and Richards, 2002; Goins et al., 1981]. We will take advantage of the overlap of LP and SP frequency band and numerically combine the two data streams to obtain a single broadband data stream and complete continuous spectra that cover both frequency bands of LP and SP data. This will enable us to carry out the spectral analysis with the wider frequency band necessary to improve the estimation of 


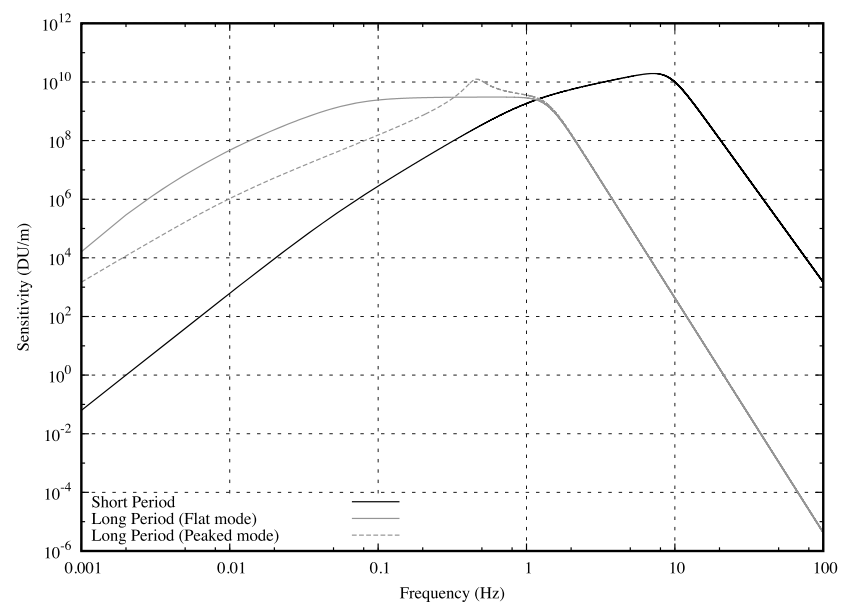

Figure 3. Instrument response of Apollo seismometers of long and short periods. The black curve refers to the short periods, and the gray curves refer to the long-period seismometer, respectively. Since the long-period seismometer had two observation modes (flat and peaked modes), two curves are shown for the long-period seismometer. The bold and dashed curves represent the flat and broad modes, respectively. corner frequency and seismic moment. We do this through a least squares method, by finding the best spectrum $S(\omega)$ that minimizes residual $l$,

$$
\begin{aligned}
I= & \frac{\left\|d_{L P}(\omega)-T_{L P}(\omega) S(\omega)\right\|^{2}}{\sigma_{L P}^{2}(\omega)} \\
& +\frac{\left\|d_{S P}(\omega)-T_{S P}(\omega) S(\omega)\right\|^{2}}{\sigma_{S P}^{2}(\omega)}
\end{aligned}
$$

where $d_{L P}(\omega)$ and $d_{S P}(\omega)$ are the data from LP and SP seismometers, respectively; $T_{L P}(\omega)$ and $T_{S P}(\omega)$ are the transfer functions; $\sigma_{i}^{2}$ represents the instrument noise; $\omega$ is the frequency; and the \|\| indicates the absolute value. The noise was estimated from the data with no seismic events, corresponding to the data just before the examined event. The time window was fixed to the same value as the window used to evaluate the signal spectra (typically 30-60 s) and data about 5-10 min before the signal was used for the noise evaluation (data without gaps, glitch, or spikes were chosen). We used signal arrival from the moonquake catalog on Nakamura [1992], and this was used as reference time throughout the analyses. For the best $S(\omega)$, we need $\frac{d l}{d S}=0$.

$$
\begin{aligned}
\frac{d l}{d S} & =\frac{d}{d S}\left\{\frac{\left(d_{L P}(\omega)-T_{L P}(\omega) S(\omega)\right)-\left(d_{L P}(\omega)-T_{L P}(\omega) S(\omega)\right)}{\sigma_{L P}^{2}(\omega)}+\frac{\left(d_{S P}(\omega)-T_{S P}(\omega) S(\omega)\right)-\left(d_{S P}(\omega)-T_{S P}(\omega) S(\omega)\right)}{\sigma_{S P}^{2}(\omega)}\right\}(2) \\
& =\left(\frac{\left\|T_{L P}(\omega)\right\|^{2}}{\sigma_{L P}^{2}(\omega)}+\frac{\left\|T_{S P}(\omega)\right\|^{2}}{\sigma_{S P}^{2}(\omega)}\right) S(\omega)-\frac{T_{L P}(\omega)-d_{L P(\omega)}}{\sigma_{L P}^{2}(\omega)}-\frac{T_{S P}(\omega)-d_{S P(\omega)}}{\sigma_{S P}^{2}(\omega)}
\end{aligned}
$$

The bar above $d_{i}(\omega), T_{i}(\omega)$, and $S(\omega)$ refers to the complex conjugate. This gives us

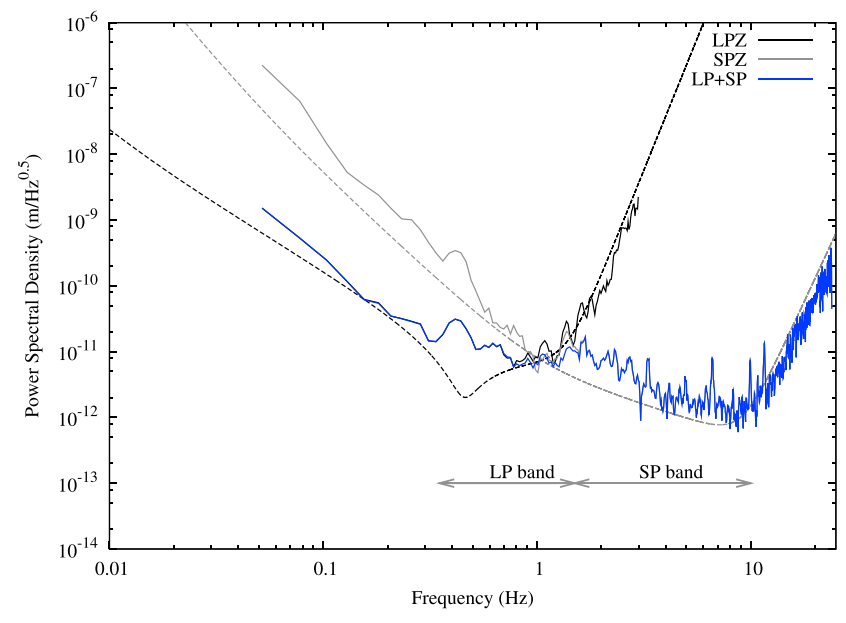

Figure 4. Example of the hybrid broadband spectrum composed of LP and SP combined data. The plot shows the combined spectrum from deep moonquake event A06 (20 May 1976, 17:35). Spectra from the LP and SP narrowband seismometers are shown together with the hybrid broadband spectrum for comparison. The dashed line shows the noise level of the seismometer. Outside the frequency bands, the obtained spectra are comparable to the instrumental noise and show similar trends.
$\frac{S(\omega)={ }^{T_{L P}(\omega)-d_{L P(\omega)} / \sigma_{L P}^{2}(\omega)}+{ }^{T S P}(\omega)-d_{S P(\omega)} / \sigma_{S P}^{2}(\omega)}{\left\|T_{L P}(\omega)\right\|^{2} / \sigma_{\sigma_{L P}^{2}(\omega)}+\left\|T_{S P}(\omega)\right\|^{2} / \sigma_{S P}^{2}(\omega)}$

An example of a combined spectrum is shown in Figure 4. The high-frequency components and low-frequency components are mainly constrained with SP and LP spectra, respectively, and we can see that the combined spectrum covers the frequency band of the two seismometers. To run the combination, the LP and SP seismometers need to be aligned and be on the same axis. Since only the vertical $(Z)$ axis is available for the $S P$, this approach is only possible on the vertical axis. Furthermore, since there were some malfunctions on SPZ and LPZ axes for station 12 and station 14, this processing is possible only with data from stations 15 and 16 (Figure 1). 


\subsection{Spectral Parameters and Source Time Function}

For the source time function $A^{\text {source }}(f)$, we use $\omega^{2}$ model, which is a well-accepted model in terrestrial seismology [Aki and Richards, 2002] expressed as

$$
A^{\text {source }}(f)=\frac{\Omega_{0}}{1+\left(f / f_{c}\right)^{2}}
$$

where $f_{c}$ is a corner frequency and $\Omega_{0}$ is the DC value of the spectrum. This shows that the seismic spectra are flat up to the corner frequency and rolls-off by the power of 2 at higher frequencies. The $\omega^{2}$ model was developed for far-field displacement excited by a slip on a fault, which assumes a double-couple force as the seismic source. The model adopts the Haskell fault model where a rupture is expressed as a boxcar function whose width is given by the risetime and the duration of the rupture. The simple fault model predicts a spectral feature as described in the $\omega^{2}$ model and it explains terrestrial observations very well [see reviews, e.g., Aki and Richards, 2002; Shearer, 2009, and references therein]. In addition to the source time function, seismic spectra contain the information on the effects of propagation and site reverberation, which are observed in the form of intense scattering and a strong coda of the seismogram [Aki and Richards, 2002]. In order to account for these effects, we express the observed spectrum $A^{\text {source }}(f)$ based on a theoretical model as follows:

$$
A(f)=A^{\text {source }}(f) \times \exp \left(-\frac{\pi f t}{Q}\right) \times R_{\text {local }} \times R_{\text {raypath }}+\text { noise }
$$

The first term is the source time function described previously. The second term accounts for the anelastic attenuation, where $Q$ is the attenuation factor and $t$ is the travel time. $R_{\text {local }}$ and $R_{\text {raypath }}$ represent the local effect or station function and the effect depending on the raypath between the source and the station.

\subsection{Spectral Stacking}

For better estimation of the source parameters, the nonseismic source term should be well constrained. While the source time function varies for each event, the nonseismic source component can be regarded as being almost identical when we fix a pair of station and seismic sources. Since deep moonquakes occur repeatedly at certain nests, we were able to stack the data to evaluate the nonseismic source component. First, a tentative corner frequency $f_{c}$ and tentative $D C$ value $\Omega^{\prime}{ }_{0}$ are assigned to each event

$$
A_{i}(f)=A_{i}^{\text {source }}\left(f ; \Omega^{\prime}{ }_{0}, f^{\prime}{ }_{c}\right) \times \exp \left(-\frac{\pi f t}{Q}\right) \times R_{\text {local }} \times R_{\text {raypath }}+\text { noise }
$$

where $i$ represents the $i$ th event for a station-seismic source pair. With the tentative value, we calculated the source time function and eliminate the term by dividing the spectrum with the source time function, leaving only the source-station-dependent term and the noise on the left hand.

$$
\left.A_{i}(f)\right|_{A_{i}^{\text {source }}\left(f ; \Omega_{0}^{\prime}, f_{c}^{\prime}\right)}=\exp \left(-\frac{\pi f t}{Q}\right) \times R_{\text {local }} \times R_{\text {raypath }}+{ }^{\text {noise } / A^{i \text { source }}\left(f ; \Omega_{0}^{\prime}, f_{c}^{\prime} c\right)}
$$

By stacking this for all events for a certain pair of source and station, we can extract the source-stationdependent term. Statistically speaking, we can expect that the noise will be reduced by the root square of the number of the events stacked, if we assume that the noise is purely random [e.g., Baker, 1999]. After stacking $n$ events, we obtain

$$
\frac{1}{n} \Sigma^{A_{i}(f)} /_{A_{i}^{\text {source }}\left(f ; \Omega^{\prime} 0, f_{c}^{\prime}\right)}=\exp \left(-\frac{\pi f t}{Q}\right) \times R_{\text {local }} \times R_{\text {raypath }}+\frac{1}{n} \Sigma^{\text {noise } / A A^{\text {source }}\left(f ; \Omega_{0}^{\prime}, f_{c}^{\prime}\right)}
$$

where it is expected that $\frac{1}{n} \sum^{\text {noise }} / A^{\text {source }}\left(f ; \Omega^{\prime}{ }_{0}, f^{\prime} c\right) \ll \exp \left(-\frac{\pi t t}{Q}\right) \times R_{\text {local }} \times R_{\text {raypath }}$. The right-hand term can be regarded as the response between the source and the station including all the nonseismic source effects, such as local effects during the propagation or noise and spikes of instrumental origin. This response was used to correct the observed spectrum so that we can extract the source time function from the data. Using this corrected spectrum, we reestimate $f_{c}$ and $\Omega_{0}$. By running this process iteratively, we can refine the estimation of $f_{c}$ and $\Omega_{0}$. For the first iteration, we need to give some tentative values for $f_{c}$ and $\Omega_{0}$ as initial values, which were expressed as $f_{c}$ and $\Omega_{0}^{\prime}$ above. 

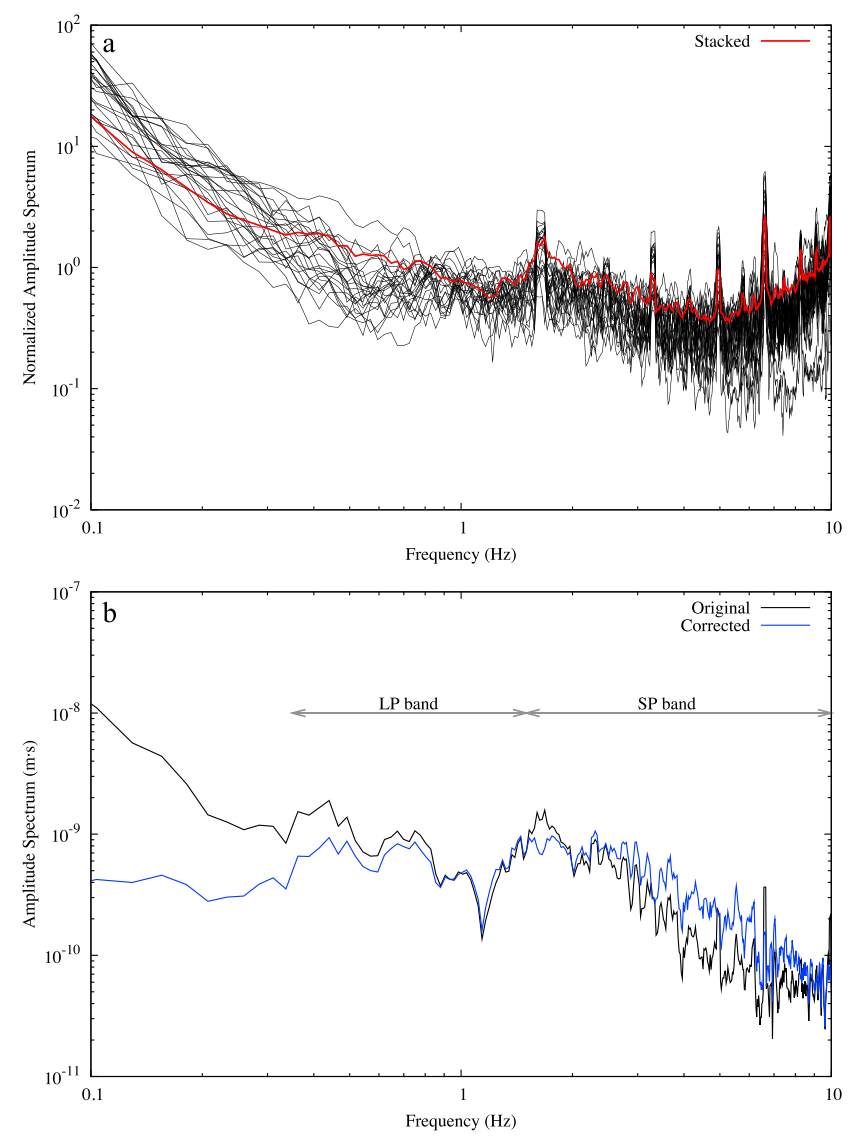

Figure 5. Stacking of background spectrum and background correction. (a) All the individual spectra (black) and the stacked spectrum (red). We see that peaks and spikes that are common among the spectra are also present in the stacked spectrum. (b) An example of the correction carried out for the seismic spectrum. Spikes (e.g., 1.5, 5, and $7 \mathrm{~Hz}$ ) are removed by the correction and attenuation is also corrected with the data processing.
We started the inversion with initial values $f_{c}$ and $\Omega_{0}^{\prime}$, estimated with a fixed $Q$ model given in the previous study [Nakamura and Koyama, 1982], which gives about $Q=2000$ 3000. After the first iteration, we stack the spectra with the source time function component removed to estimate the background spectra as described previously (Figure 5 and equation (8)). We, in turn, take the original spectrum $A_{i}(f)$ and correct this with the background spectra obtained from the stack where we expect only the source time function to be contained in the corrected spectra (Figure 5). With the corrected spectra, we reestimate $f_{c}$ and $\Omega_{0}$ with the least squares fitting of the source time function $A^{\text {source }}(f)$. This will give us new sets of $f_{c}$ and $\Omega_{0}$. With a new set of $f_{c}$ and $\Omega_{0}$ in hand, we reexecute the stack to improve the estimate for the background spectrum. The process will be iterated until the improvement of the iteration is smaller than the error bar. The iteration converges typically within 5-10 iterations. Figure 5 shows an example of spectral stacking and correction using the background spectrum.

\subsection{Correction of Scattering Effect}

Intense scattering is one of the characteristic features of lunar seismograms, and a significant amount of energy is redistributed into its coda [Dainty et al., 1974]. This point needs to be taken into account for estimation of the seismic moment. A previous study [Goins et al., 1981] treated this problem by introducing a correction factor estimated from amplitude decay of coda. We took a similar approach and applied a correction factor to our estimation. Figure 6 shows the absolute value of the amplitude of the largest A06 event observed at the station 16. The blue shaded portion of the waveform refers to the time window we used to estimate $f_{c}$ and $\Omega_{0}$. We expect that the signal is less contaminated by reflected and scattered signal at its first arrival. We therefore used the first data strip and correct for the redistribution of the energy using the following process. We divided the seismic signal into smaller segments with the same width as the reference time windows. Figure 6 demonstrates an example of data segments used in the analyses. The blue shaded segment of the data, which starts from the reported signal arrival [Nakamura, 1992], was first used to estimate $f_{c}$ and $\Omega_{0}$. Then, a Fourier transform was applied on each data segment and the $\Omega_{0}$ was estimated for each data segment. The time variation of $\Omega_{0}$ is shown in Figure 6. This time variation of $\Omega_{0}$ was used to correct the seismic moment that was evaluated with the first segment. The variation of $\Omega_{0}$ shows good correlation with the amplitude of the waveform and the signal level exceeds the noise level 300-800 s after the signal start. We assume that $\Omega_{0}$ degrades linearly and express the variation of $\Omega_{0}$ with time with a linear function by fitting the data. We assume that the seismic energy is contained in the seismic signal until the $\Omega_{0}$ reaches 0 . In other words, we assume that the seismic energy is redistributed inside the triangle bounded by $x$ axis, signal arrival, and the degrading function of $\Omega_{0}$ estimated previously. This triangle is shown as a 

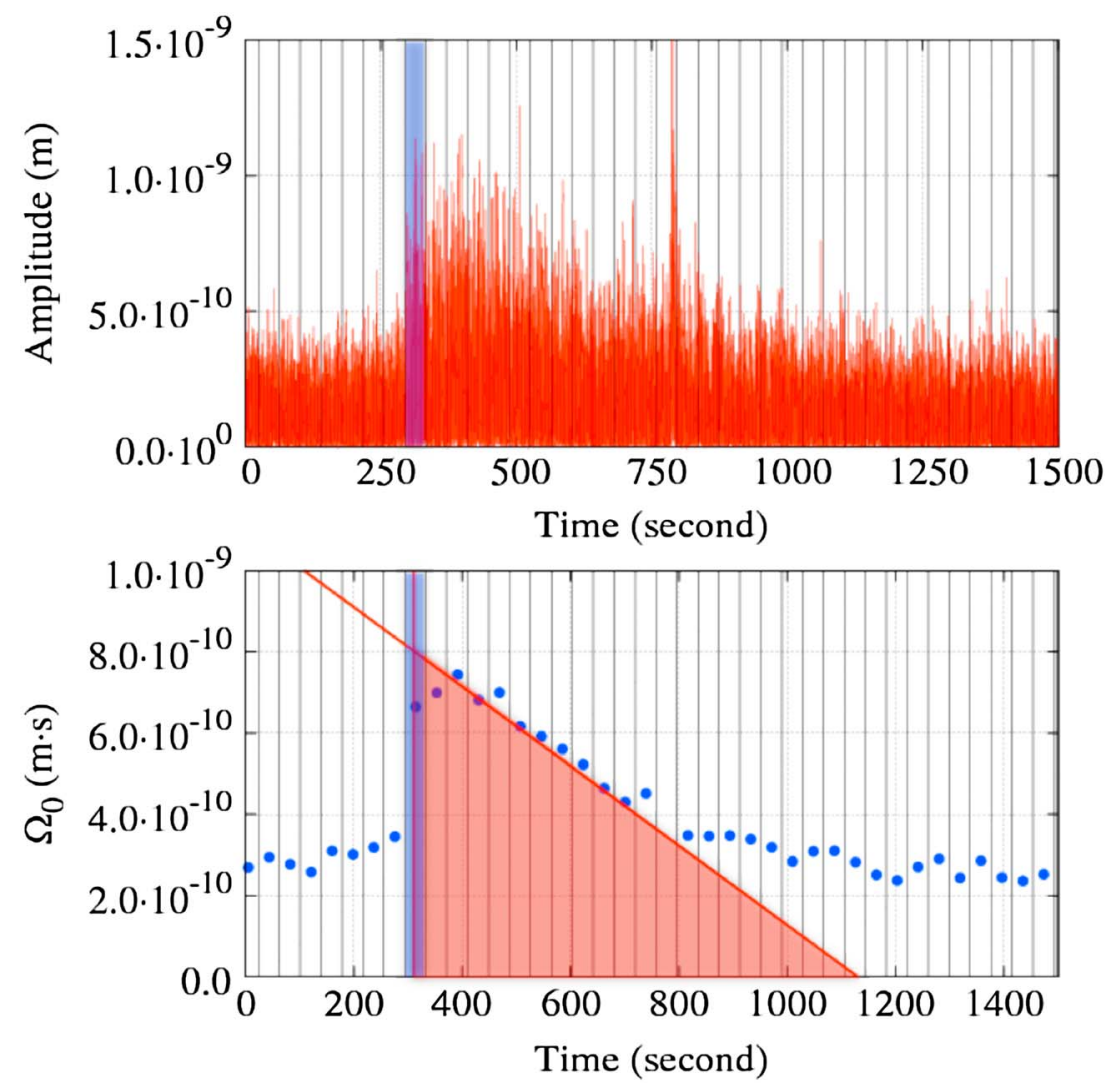

Figure 6. An example of (top) amplitude variation and the (bottom) time variation of $\Omega_{0}$, which is the DC value of the spectrum, for the A06 deep moonquake event. The blue shaded region shows the short time window used to estimate $f_{c}$ and $\Omega_{0}$. The data were divided into short strips of data $40 \mathrm{~s}$ long. The data strip is divided by a black line in the figure. In the bottom plot, $\Omega_{0}$ estimated for each data strip is shown in blue dots. The time variation of $\Omega_{0}$ was fitted with the red line in the plot. The red triangle shows the portion of the signal carrying the scattered seismic energy. The plot uses an arbitrary time origin.

red triangle in Figure 6. By comparing the total energy within the triangle and energy within the first data segment of the seismic wave, we can evaluate the correction factor $\alpha$. This can be expressed as

$$
\alpha=\frac{\sqrt{\sum \Omega_{0, i}^{2}}}{\Omega_{0,1}}
$$

where $\Omega_{0,1}$ refers to $\Omega_{0, i}$ of the ith data segment from the signal arrival. The characteristics of the coda or the degrading feature of the $\Omega_{0}$ are expected to be dependent of the scattering feature near the station and the raypath between the source region and the station. This means that the correction factor is a raypathdependent term (i.e., constant for a source region and station pair) and not an event-dependent term. Thus, we calculated the correction factor for each set of source region and station.

\section{Estimation of Source Parameters}

\subsection{Spectral Fitting and Evaluation of Spectral Features}

After having processed the series of data we expect that the spectra contain only the source time function and we fit the data to the $\omega^{2}$ model described previously. Through the fitting, we obtain $f_{c}$ and $\Omega_{0}$. For the events detected at multiple stations, we carry out the fitting for the two stations simultaneously since $f_{c}$ and $\Omega_{0}$ should be identical for the same event. We did the above analysis for 131 events from three deep moonquake nests A01, A06, and A07, which are some of the most active deep moonquake nests observed so far (Figures 1 and 7). While more than 100 source regions have been identified [Nakamura, 2003], we focus on the three active nests, which should give the best quality data set for our first attempt to carry out 


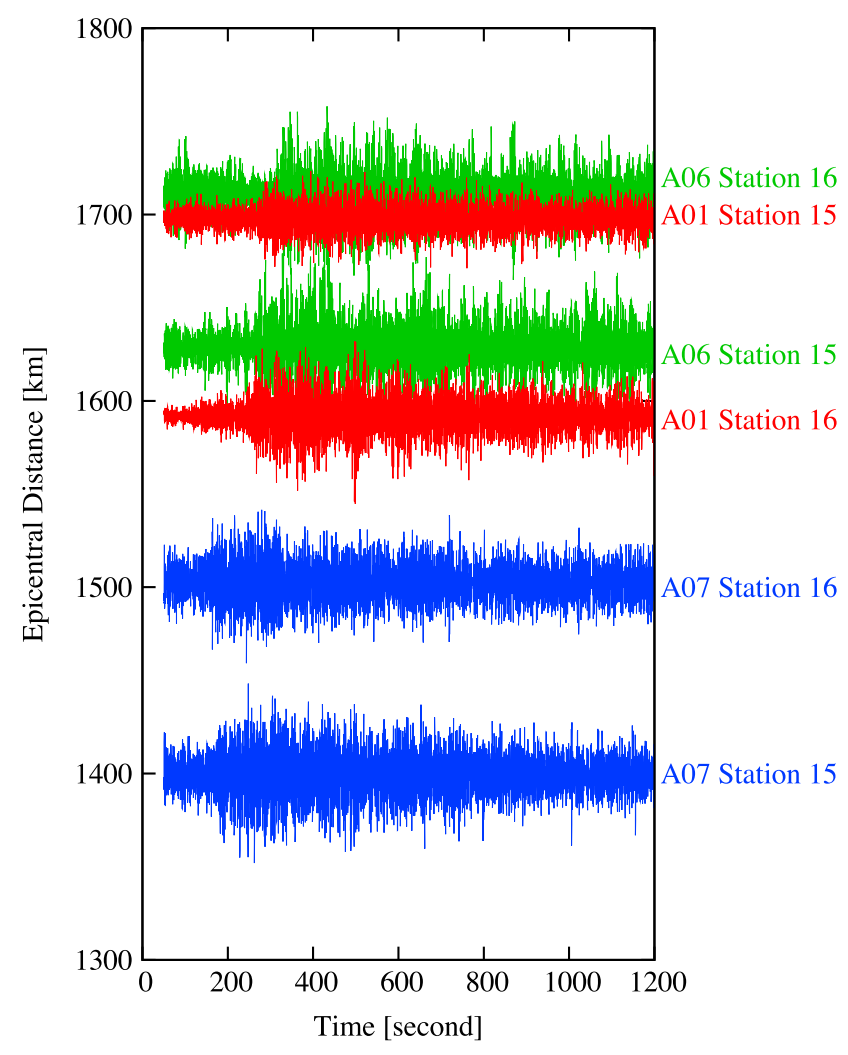

Figure 7. A01, A06, and A07 deep moonquake events. The figure shows the associated virtual broadband seismogram at each station for a single deep moonquake event, from each nest (A01: 27 March 1976, 18:48; A06: 25 November 1975, 23:20; and A07: 11 April 1976, 12:51). Note that since the seismograms refer to different events for different nests, the amplitude and arrival times are set with arbitrary offsets and amplifications are not to scale.

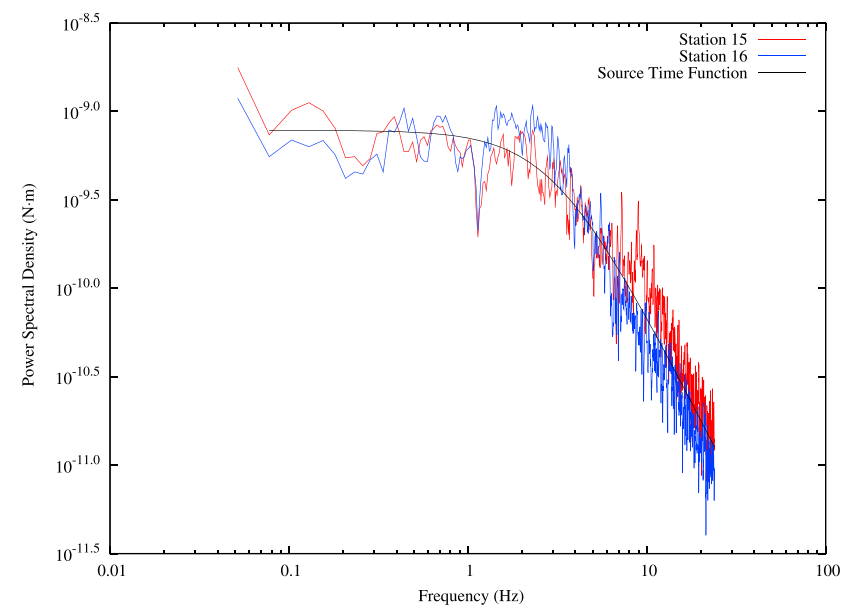

Figure 8. An example of a spectrum's fit for a seismic source function from A06 deep moonquake (20 May 1975, 17:35). The seismic spectra were corrected for attenuation and other nonseismic components (see text for details). The best fit model is shown in the plot and corresponds to a moment of $2.0 \pm 0.2 \times 10^{13} \mathrm{Nm}$ (moment magnitude $=2.8$ ) and a cutoff frequency of $3.5 \mathrm{~Hz}$. broadband spectral analysis on the Moon. The A01 nest is the most active deep moonquake source and A06 and A07 are sources that are located close to stations 15 and 16 (Figure 1). These data should be the best data set to carry out our first analysis since they have likely the best signal-to-noise ratio. Figure 8 shows the result of spectral fitting of the source time function to the data. We see that the model is in agreement with the observations and that the observations at two stations also compares favorably.

Figure 9 shows the estimated corner frequencies and seismic moments for all studied events from these three nests. By expanding the frequency range, we succeeded in estimating source parameters, not only for various nests but also over a wide range of seismic moments, ranging from $10^{12.5} \mathrm{Nm}$ to almost $10^{14} \mathrm{Nm}$, which correspond to a moment magnitude from 2.3 to 3.3. The estimated corner frequencies are distributed within a wider range but we rejected the values outside the noise roll-off of the SP $(8 \mathrm{~Hz})$. While the previous study obtained $1 \mathrm{~Hz}$ for the corner frequency, most of our corner frequencies are distributed between 1 and $8 \mathrm{~Hz}$, which is reasonable since we are investigating events of smaller magnitude.

\subsection{Estimation of Stress Drops}

Investigation of fault activities and source mechanisms in terrestrial seismology derived a scaling law between seismic moments, corner frequencies, and stress drops, which explains terrestrial observations well [Aki and Richards, 2002; Goins et al., 1981]. This is expressed as

$$
\Delta \sigma=12 M_{0}\left(\frac{f_{c}}{\beta}\right)^{3}
$$

where $\Delta \sigma$ is the stress drop, $M_{0}$ is the seismic moment, and $\beta$ is the shear velocity at the source region. Assuming that deep moonquakes are also fault activities that can be expressed with a double-couple force, which is supported by the clear appearance of $P$ and $S$ wave signals, the same scaling law should be applicable to deep moonquakes. This 
was the same assumption made in the previous study [Goins et al., 1981], and we will use the same approach to estimate the stress drops. Terrestrial observations suggest that the stress drop is independent of seismic moment and is almost constant over a wide range of seismic moments [Ide et al., 2003]. This is known as the self-similarity of seismic events. Referring to the terrestrial examples and given that the deep moonquakes occur in relatively well-defined source regions, we assume that events from the same nest occur in very similar condition, possibly on the same fault plane. Thus, we assume that stress drop is constant within a nest. This allows us to estimate the stress drop for each deep moonquake nests from the sets of $f_{c}$ and $\Omega_{0}$ we obtained previously. The scaling law (equation (10)) was fitted to the $f_{c} s$ and $\Omega_{0} s$ to obtain the stress drop. The errors on stress drop will be evaluated through the misfit. The fit was weighted by estimation error of the corner frequencies. With this assumption, we estimated stress drops of the deep moonquake nests as follows: $\mathrm{A} 01: 0.14 \pm 0.01 \mathrm{MPa}, \mathrm{A} 06: 0.09 \pm 0.01 \mathrm{MPa}$, and A07: $0.09 \pm 0.008 \mathrm{MPa}$. These stress drops are significantly larger than those estimated in the previous study [Goins et al., 1981]. This discrepancy is retrieved for the single event used in the previous study, an A01 deep moonquake event on 29 October 1975. While both studies have similar seismic moments $\left(6.8 \times 10^{13} \mathrm{Nm}\right.$ in our study and $7.4 \times 10^{13} \mathrm{Nm}$ in the previous study), the corner frequency obtained here is higher $(2.4 \pm 0.1 \mathrm{~Hz}$ in our study and $1 \mathrm{~Hz}$ in the previous study) which resulted in a higher stress drop. This is reasonable since the seismic moment is mainly constrained by the long-period component of spectra while corner frequency is constrained by the high-frequency component where we see the improvement with our broadband data.

\section{Discussion}

\subsection{Self-Similarity of Seismic Activities and Fault Roughness}

While the least squares fit performed in the previous section enables us to estimate stress drops of deep moonquakes, the model did not result in a satisfactory fit especially at low and high frequencies. Indeed, the trend of the data appears to be smaller than the -3 slope, which has been suggested from traditional scaling laws for $f_{c}, M_{0}$, and $\Delta \sigma$ [e.g., Aki and Richards, 2002; Goins et al., 1981]. This implies that the deep moonquakes are not self-similar where stress drops are independent of seismic moments [Ide et al., 2003] and stress drops show some moment dependency.

Self-similarity of quakes and moment dependency of stress drops have been debated for a long time [e.g., Ide et al., 2003; Allmann and Shearer, 2009; Abercrombie, 2014]. While many studies claim that earthquake stress drops are independent of their seismic moments [e.g., Ide et al., 2003], the estimated stress drops have a large scatter of 0.1-100 MPa. This scatter is interpreted as unmodeled sources of error such as near-surface attenuation or directivity effects [Candela et al., 2011], but some studies claim that heterogeneous properties of faulting and fault surface influence the values of static stress drop [Allmann and Shearer, 2009; Mayeda and Walter, 1996].

One of the key properties affecting the moment dependency of stress drop is the fault roughness. The classical view of the fault roughness is that the fault roughness is also self-similar, where the vertical and horizontal dimension is amplified isotropically regardless of the scaling transformation. In other words, the roughness properties are the same for microscopic and macroscopic scales. Such self-similar faults result in self-similarity of quakes and stress drops are independent of seismic moments. Many studies regarded that the fault surface can be characterized by a self-similar roughness.

On the other hand, observations of actual fault surface imply that the fault roughness is better expressed as a self-affine surface rather than a self-similar surface [Schmittbuhl et al., 1993; Candela et al., 2012; Bistacchi et al., 2011]. When the fault roughness is self-affine rather than self-similar, the vertical and horizontal dimension will not be amplified isotropically. In this case, the roughness defined at microscopic scale may be different at macroscopic scale. By applying a self-affine model, we would be able to take into account the evolution of fault roughness for different fault sizes. Since the seismic moment is a product of fault size, average slip, and shear modulus of the fault [Aki and Richards, 2002], self-affinity of fault and different roughness features at microscopic and macroscopic scales will have significant influence on the moment dependency of stress drops. Interestingly, while observations of faults surface exposed as an outcrop is better represented as a self-affine roughness, fault surface at seismogenic depth observed with radar sounder also supports the self-affine surface for seismic faults [Bistacchi et al., 2011]. This implies that the self-affine geometrical model may represent a global feature of natural fault surfaces. Schmittbuhl et al. [2006] proposed a 

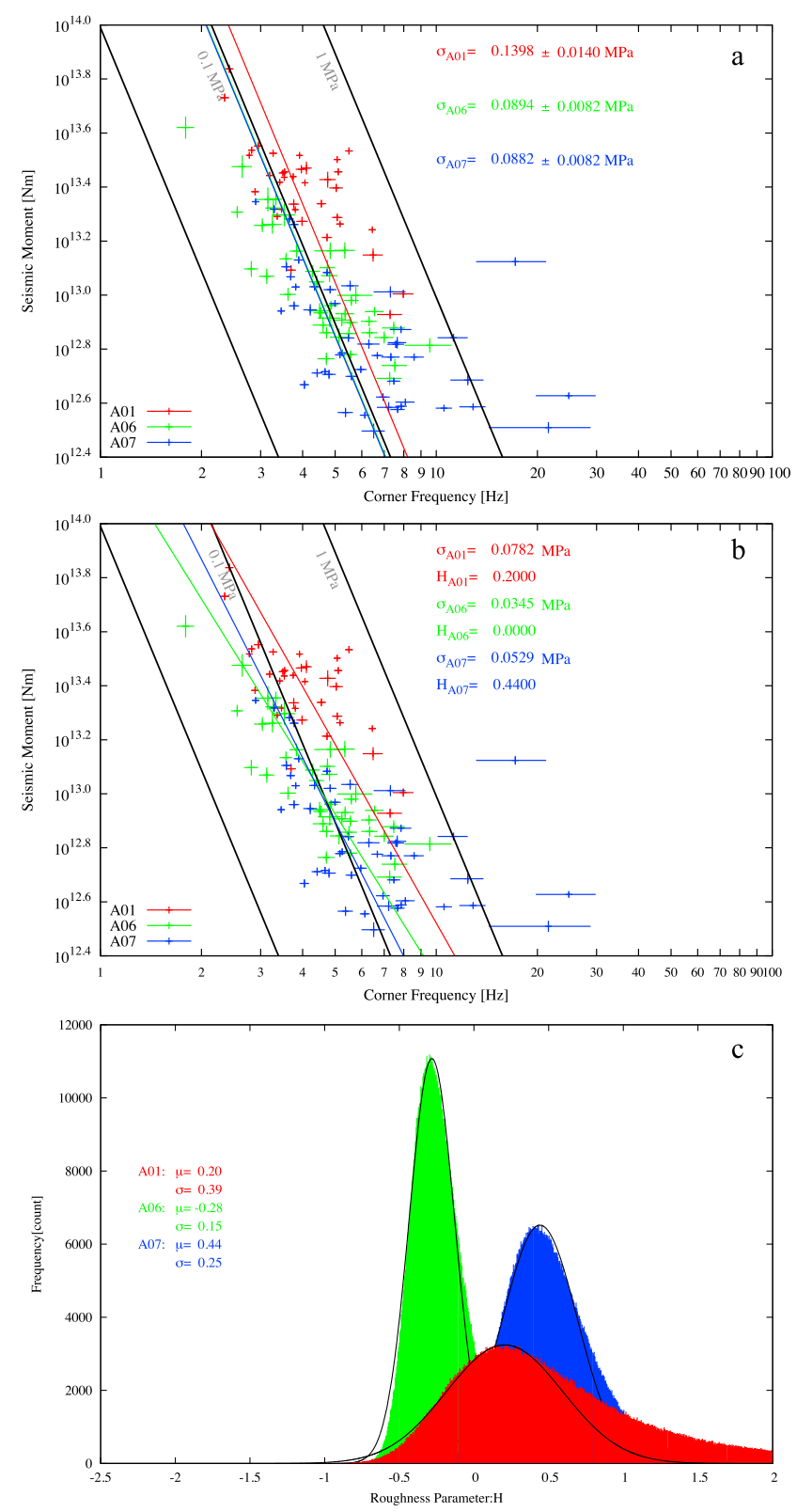

Figure 9. Source parameters estimated for A01, A06, and A07 deep moonquake nests. Corner frequencies and seismic moments estimated for each event from the three nests we investigated are shown in the plot as A01: red, A06: green, and A07: blue. The best fit model obtained and the corresponding stress drops are also shown. Figure 9a shows the results for a self-similar fault, and Figure 9b shows the results for a self-affine fault including effect of roughness. Figure $9 \mathrm{c}$ shows the distribution of roughness parameter $H$, as described in the text, obtained in each subset of bootstrap resampling. The black line refers to the Gaussian distribution that was fitted to each histogram whose expected values $\mu$ and standard deviations $\sigma$ are also shown. The $\mu$ can be regarded as the most probable value of $H$ with an error quantified by $\sigma$. For all nests, the fits prefer low $H$ and low stress drops are preferred. fault model taking into account the effect of such a self-affine fault surface. This model explains well the stress field observed before and after the Nojima Fault of Japan [Schmittbuhl et al., 2006]. Candela et al. [2011] took this model to investigate the effect of a self-affine fault surface on stress drops and suggested that this will result in moment dependency of the stress drop.

\subsection{Self-Affine Fault Roughness and Seismic Moment Dependency of Stress Drop}

In the self-affine fault model, the fault's roughness can be smoother at large scale, while at small scale, there is a large variety of patterns of smallamplitude asperities [Candela et al., 2011]. The affinity of the fault is expressed through the Hurst exponent $H$, which is also known as a roughness parameter. In the selfsimilar model, when the horizontal dimension is amplified from $\delta x$ to $\lambda \delta x$, the vertical roughness will also be amplified from $\delta y$ to $\lambda \delta y$. For a selfaffine model, when the horizontal dimension is amplified from $\delta x$ to $\lambda \delta x$, the vertical roughness will be amplified from $\delta y$ to $\lambda^{H} \delta y$. Thus, when $H=1$, it will be identical to be a selfsimilar model. On the other hand, for smaller $H$, the fault roughness flattens with increasing dimensions. Candela et al. [2011] claims that this will result in stress drop variation with fault size, $\Delta \sigma \propto r^{H-1}$, where $r$ is the fault size [Candela et al., 2011]. We follow their discussion and assume that $\Delta \sigma=\Delta \sigma_{0}\left(r / r_{0}\right)^{H-1}$, where $r_{0}$ is a reference fault size where we define the reference stress drop.

Average stress drop and seismic moments are expressed as follows with the traditional model [Aki and Richards, 2002]:

$$
\Delta \sigma=\frac{7 \pi}{16} G \frac{a}{r} M_{0}=G A a
$$

where $G$ is the shear modulus, $a$ is an average slip, $r$ is the size of fault, and $A$ is an area of rupture. When we assume a circular fault, as is often the case for terrestrial seismology [Aki and Richards, 2002], we get 


$$
\Delta \sigma=\frac{7}{16} \frac{M_{0}}{r^{3}}=12 M_{0}\left(\frac{f_{c}}{\beta}\right)^{3} \Delta \sigma_{0}\left(\frac{r}{r_{0}}\right)^{H-1}=12 M_{0}\left(\frac{f_{c}}{\beta}\right)^{3} \Delta \sigma=12 M_{0}\left(\frac{f_{c}}{\beta}\right)^{3}\left(\frac{r_{0}}{r}\right)^{1-H}
$$

Introducing such relations, we are able to take into account the fault roughness and moment dependency of stress drop. The parameter used in this study refers to the values by Goins et al. [1981], which is $\beta=4.2 \mathrm{~km} / \mathrm{s}$. We referred to a previous study that estimated the size of the A01 deep moonquake nest [Nakamura, 1978] to define the reference fault size $r_{0} \sim 1 \mathrm{~km}$. However, little is known about the fault size of deep moonquakes for the moment. The small seismic moment we obtained in this study implies either a small fault area and/or small slip. When we assume a shear modulus of 60 GPa for the deep moonquake source regions and from the obtained seismic moment, which is $10^{12}-10^{14}$, we obtain a slip of $10^{-5}-10^{-2} \mathrm{~m}$ for a $1 \mathrm{~km}$ circular fault. If the slip of deep moonquakes is larger, the fault size can be smaller, and the effective stress drop for each seismic event can be larger.

Figure 9 shows results obtained with the self-affine model. As was done for a self-similar model, we carried out least squares fitting with the self-affine model to estimate the stress drops. Here we used equation (12) instead of equation (10) for the fitting. As shown in Figure 9, the misfit of the least squares fit is improved by introducing roughness parameter $H$ to the stress drop estimation. This implies that our data supports the self-affine fault model for deep moonquakes.

However, the small amplitudes of the recorded quakes lead to large scatters in the data set, leaving significant uncertainties in our estimation. Such scatters in the data may result in erroneous $H$ value due to outliers that may not be suitable for use in the fitting. We therefore took a statistic approach to estimate $H$ and its uncertainty. Instead of defining stress drops and $H$ through single least squares fitting, we executed multiple runs of least squares fitting and studied the distribution of the $H$ value we obtained from each fitting. Each least squares fit was carried out with a data set, which was resampled from the original data set. This was done with a statistical method known as bootstrapping [Efron, 1979]. This will enable us to avoid misinterpretation of the data from some outliers, since the random process will generate subsets with and without the outliers. If a few outliers were contaminating our least squares fit, it is more likely that the outliers will not be included in the resampled data set and the result with the outliers will be discarded in the following statistical analysis. The fit was weighted by estimation error of the corner frequencies. Statistically speaking, the distribution of $H$ obtained from the multiple resampling and fitting should give us representative $H$ values for the data set.

We carried out one million resamplings and fittings and studied the distribution of the resultant $H$ values. From the histograms of the $H$ values, the mode should be regarded as the representative $H$ and the standard deviation can be regarded as the deviation of $H$ value due to the scatter in the data set. Figure 9 also shows the histogram for $\mathrm{H}$ obtained for each bootstrapping subset. Assuming that the histogram follows a Gaussian distribution $\left(f(x)=1 / \sqrt{2 \pi \sigma^{2}} \exp \left(-(x-\mu)^{2} / 2 \sigma^{2}\right)\right)$, the mode $(\mu)$ will correspond to the $H$ value and the standard deviation $(\sigma)$ can be regarded as the estimation error. A similar statistic approach is also possible for the stress drop where we can define stress drop from the histogram. By definition, the stress drop we obtained here corresponds to the value that is obtained with the representative $H$ value, which is also defined statistically. For all nests, we obtained $H$ values that are significantly lower than 1 , which implies that deep moonquake faults are better explained with a self-affine model. This means that the fault roughness flattens with fault size and the fault can be considered to be smooth in macroscopic scale. The sets of $H$ and stress drop $(\Delta \sigma)$ that give the best fit for each nest are as follows: A01: $H=0.20 \pm 0.39, \Delta \sigma=0.06 \pm 0.02 \mathrm{MPa}$; A06: $H=-0.28 \pm 0.15, \Delta \sigma=0.025 \pm 0.005 \mathrm{MPa}$; and A07: $H=0.44 \pm 0.25, \Delta \sigma=0.04 \pm 0.01 \mathrm{MPa}$. In the case of A06, the Hurst exponent attains negative values but this does not oppose the tendency that the deep moonquake faults are smooth with low $H$ values. For the smooth faults, the stress drops are lower than for the self-similar model and are comparable to the tidal stress for all deep moonquake nests.

The range for the roughness $H$ values of terrestrial faults is 0.5-0.8 [Candela et al., 2011; Candela et al., 2012]. Thus, our results imply that the deep moonquake faults are smoother compared to terrestrial faults in terms of the Hurst exponent. While studies suggest evolution of fault surface roughness with maturity, there has been no evidence of variation in $H$ values with maturity [e.g., Brodsky et al., 2011; Brodsky et al., 2015]. Deep moonquake faults that have been active monthly since their formation can be regarded as very active faults compared to terrestrial faults. This major aging difference as compared to the Earth's much younger 
faults support a very developed abrasion of the fault surface through the repeated quakes (A1 has typically a rate of about 80 events per year [Weber et al., 2009]). Qualitatively speaking, it is reasonable to expect a very smooth fault for deep moonquakes but its implication for the Hurst exponent should be investigated more in detail in future work.

When applied to the deep moonquake faults, the self-affine fault model provides stress drops ranging from the maximum of the shear stresses generated by the tides to a fraction of these [Weber et al., 2009] and supports the hypothesis that Earth tides not only trigger deep moonquakes but also provide most of the stress glut generating the quakes. This idea is also compatible with the polarization of deep moonquake signals [Frohlich and Nakamura, 2009], which is difficult to realize with unidirectional tectonic stress. The question remains whether the tidal stress will be able to trigger a slip under a pressure of $\sim 4 \mathrm{GPa}$. Experimental studies show that the friction coefficient decreases drastically with lubrication from granular or fractured layer near fault boundary, which grows with fault activities [Collettini et al., 2009; Reches and Lockner, 2010; Di Toro et al., 2011; De Paola et al., 2011]. While quantitative evaluation is yet to be investigated, an extremely smooth fault supports the idea that tides are the dominant source of excitation of deep moonquakes.

\subsection{Brittle-Ductile Transition Temperature at Deep Moonquake Sources}

The previous discussion suggests that the tidal stress is the main excitation force of deep moonquakes. This point needs to be taken into account in evaluating the brittle-ductile transition temperature. Notably, we need to take into account the strain rate from the tides and not the tectonic strain rate. This is likely to have a significant impact on the temperature since the tidal strain rate is about 3-4 magnitudes larger than the tectonic strain rate in terrestrial seismogenic regions. This is due to the monthly periodicity of the strain variation, in contrast to the secular-tectonic strain accumulation on Earth. As observed on the terrestrial outer trench high at subduction zones, which is regarded as a fast-moving tectonically active areas with a high strain rate, a larger temperature of the brittle-ductile transition is expected for deep moonquakes [McKenzie et al., 2005].

Models were proposed to evaluate the brittle-ductile temperature for a given composition, and we are able to quantify this for a set of temperature, differential stress, and rheological parameters. The lower mantle where the deep moonquake source regions rest is likely to be an olivine-rich environment. Here we will use the model adopted in Boettcher et al. [2007] for a dry olivine single crystal, and correct it for the pressure range of the deep moonquakes. As discussed previously, the stress drop obtained from the spectral analyses implies that the tidal stress is the dominant stress source for deep moonquakes. Thus, we use parameters of tidal stress between the Earth and the Moon in the following discussion. Boettcher et al. [2007] showed that deformation of the asperities occurs according to flow laws determined from indentation creep tests on dry olivine single crystals. Following Boettcher et al. [2007], the strength of olivine $\sigma_{A}$ can be expressed as

$$
\sigma_{A}=\sigma_{P}\left[1-\left(\frac{-R T}{H} \ln \frac{\varepsilon}{B}\right)^{1 / q}\right]
$$

where the Peierl's stress $\sigma_{P}=8500 \mathrm{MPa}$, the gas constant $R=8.314 \mathrm{~J} / \mathrm{mol} / \mathrm{K}$, the activation enthalpy $H=5.4 \times 10^{5} \mathrm{~J} / \mathrm{mol}$, the empirical constant $B=5.7 \times 10^{11} \mathrm{~s}^{-1}$, the exponent $q=2$, and ${ }^{\prime} \varepsilon$ is the strain rate at the source region. This will enable us to estimate olivine strength $\sigma_{A}$ at a given temperature for a given strain rate.

Among the parameters, variables are strain rate, temperature, and strength of olivine. Since we assumed that deep moonquakes are excited by tides, strength should be comparable to tidal stress. Thus, if we can calculate the strain rate at deep moonquake regions, we can obtain the temperature and this correspond to the brittle-ductile transition temperature. Strain rate can be calculated from a given model of the interior structure. Here we used both integrated lunar structure models from Gagnepain-Beyneix et al. [2006] with the core proposed by Weber et al. [2011] and the model of Garcia et al. [2011]. We assumed the velocity structure of Gagnepain-Beyneix et al. [2006] down to the core-mantle boundary and added the core of either Weber et al. [2011] or Garcia et al. [2011] for comparison. Then we estimated the strain rate for the deep moonquake region following the discussion of Lognonné and Johnson [2007, 2015, section 10.03.2.3 and Appendix A]. A correction was made to account for the physical dispersion between wave velocities at seismic frequencies $(\sim 1 \mathrm{~Hz})$ and the tidal frequencies $(\sim 1 \mu \mathrm{Hz})$, leading to about $20 \%$ reduction of the shear modulus. This will be described in more detail later in the study. The homogenous model of Weber 


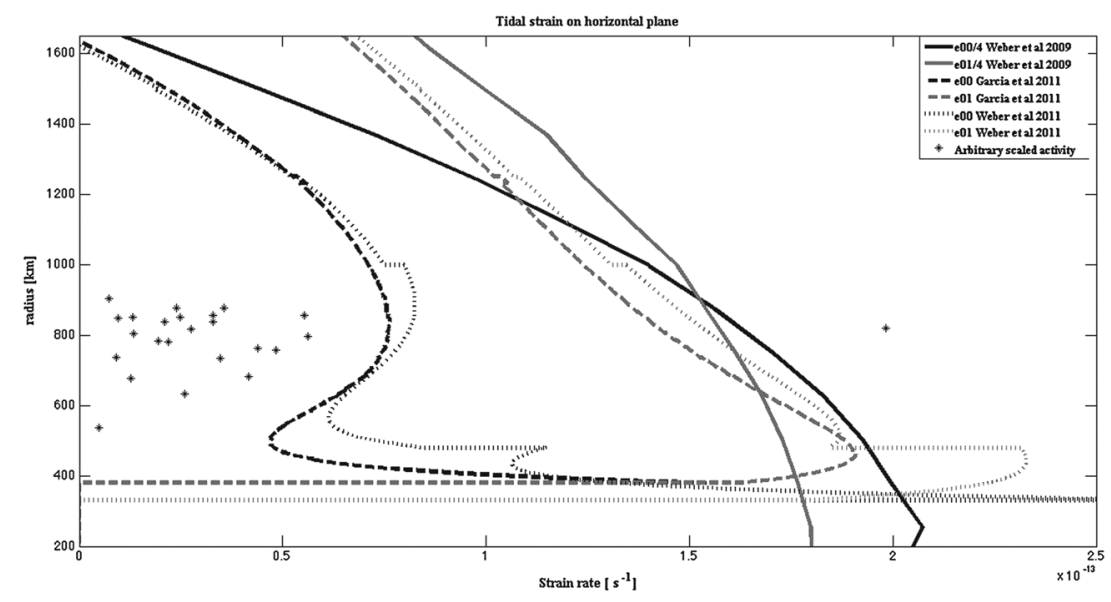

Figure 10. Comparison of two strain rate components associated with the projection of the strain tensor on a horizontal plane for the models from Weber et al. [2009], Weber et al. [2011], and Garcia et al. [2011]. The strain obtained with the model of Weber et al. [2009] has been divided by four and results from much lower values of the shear velocities than those of Garcia et al. [2011] and Weber et al. [2011]. In all cases, the strains are computed with resolution defined by the number of layers we define (512 layers, $3 \mathrm{~km}$ resolution), for the A01 deep moonquake location used by Weber et al. [2009], for an Earth location pointing toward the center of the near side of the Moon at a distance of $385,000 \mathrm{~km}$. The tidal time variation potential has been scaled in order to provide $2.5 \mathrm{kPa} / \mathrm{d}$ and $1.8 \mathrm{kPa} \mathrm{bar} / \mathrm{d}$ for the peak amplitude of the normal, respectively, shear stress variation at A1 on the horizontal plane, as illustrated by Figure 9 of Weber et al. [2009].

et al. [2009] has been also used, although the shear modulus is likely too low ( $\left.10^{10} \mathrm{~Pa}\right)$, which is equivalent to shear velocities of $1.825 \mathrm{~km} / \mathrm{s}$. The strain was estimated with a simplified tidal model described in Appendix $A$ and computed for all deep moonquakes. This results in a strain rate of about $1.5 \times 10^{-13} \mathrm{~s}^{-1}$ for the shear strain and of about $10^{-13} \mathrm{~s}^{-1}$ for the normal strain for both models of Weber et al. [2011] and Garcia et al. [2011], with respect to the horizontal plane (Figure 10). In this figure, we used the average strain of all deep moonquakes. Strains are lower by a factor of 4 than those of the low-rigidity homogenous model of Weber et al. [2009]. Note that the models of Garcia et al. [2011] and Weber et al. [2011], because of the core, reshape the profile of the strain and provide a maximum strain at the depth where we have the maximum of deep moonquake activity, as already suggested by Lognonné and Johnson [2007, 2015]. Other published models for seismic and density models provide very comparable strain rate, as long as their core radius is similar.

For each deep moonquake source region, we used the depth and specific strain rate (associated with their location) to define the shear and normal strain rate at the source region. Then the transition temperature

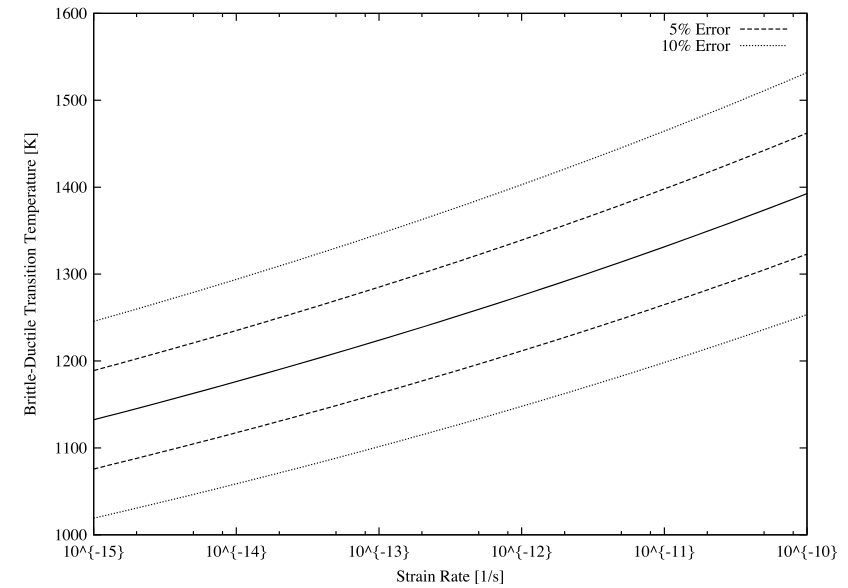

Figure 11. Variation of brittle-ductile transition temperature with strain rate and activation energy. can be obtained according to the model described with equation (13). This will give us a transition temperature as a function of depth as shown in the black triangles in Figure 11. This results in brittle-ductile transition temperatures of about 1240$1275 \mathrm{~K}$ depending on the depth of each deep moonquake source region, which ranges from 835 to $1200 \mathrm{~km}$. These values are likely to contain some errors due to the uncertainties on the parameters we used.

The brittle-ductile transition temperature obtained here is an estimation based on given condition and subject to change depending on the 

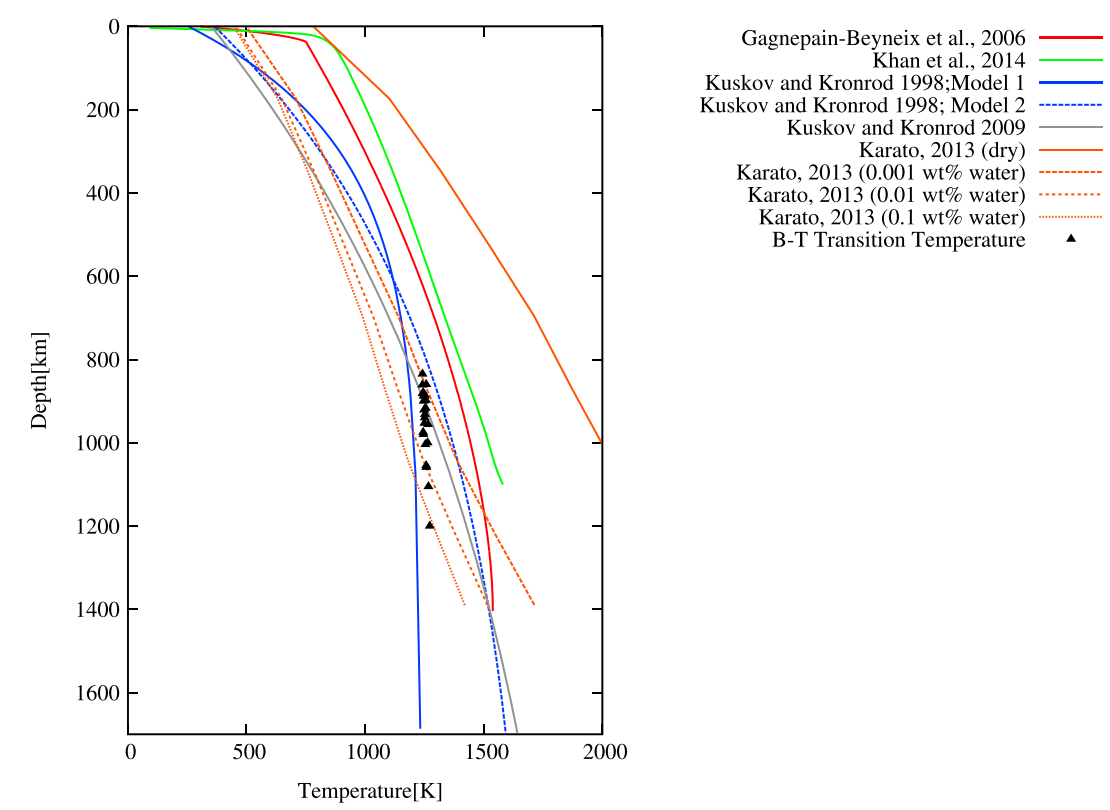

Figure 12. Comparison between temperature profiles from previous studies [Gagnepain-Beyneix et al., 2006; Khan et al., 2014; Kuskov and Kronrod, 1998; Kuskov and Kronrod, 2009; Karato, 2013] and the brittle-ductile transition temperature obtained in this study.

parameters we adopt. First, we assumed dry olivine which might be questioned given the recent discovery of water on the Moon. Studies suggest that the lunar mantle may contain about 100 ppm of water [e.g., Chen et al., 2015; Elkins-Tanton and Grove, 2011]. Experimental study on single crystal olivine for wet and dry conditions shows that strength of olivine will be factor of 1.3-1.6 weaker with water supply at $\sim 4 \mathrm{GPa}, 1200^{\circ} \mathrm{C}$ condition [Girard et al. 2013]. This implies that the effect of water of $100 \mathrm{ppm}$ is not significant for evaluation of brittle-ductile transition, especially when the differential stress or strength is expected to be small compared to Peierl's stress. Second possible source of error is the activation energy $H$ used in equation (13). Previous studies point out that activation energy of olivine has 5-10\% of uncertainties [Ohuchi et al., 2015]. This results in 50-100 K of error on the transition temperature (Figure 11). Finally, the influence of strain rate evaluation should be evaluated. Figure 11 shows the variation of brittle-ductile transition temperature with strain rate. The figure shows that replacing tectonic strain rate $\left(10^{-15}\right)$ with tidal strain rate $\left(10^{-13}\right)$ results in $100-150 \mathrm{~K}$ difference in the transition temperature. Figure 10 shows that different models result in $10^{-14}$ difference in strain rate, and this will result in tens of kelvin in the transition temperature. These are the possible source or errors and errors on the transition temperature.

\subsection{Comparing the Brittle-Ductile Transition Temperature With Temperature Models of the Moon}

The higher strain rate of tides resulted in a higher brittle-ductile transition temperature, and it is important to compare this with existing temperature profiles of the Moon. Here we referred to studies that provided temperature profile to depth of the deep moonquake source regions using variety of different approaches [Kuskov and Kronrod, 1998; Gagnepain-Beyneix et al., 2006; Kuskov and Kronrod, 2009; Karato, 2013; Khan et al., 2014]. Figure 12 shows a comparison between different temperature profiles and brittle-ductile transition temperatures obtained in the previous section. As mentioned previously, proposed temperatures in the deep moonquake source region are hotter than brittle-ductile temperatures even allowing for a higher brittle-ductile transition as temperature proposed in this study. There are two models that satisfy the temperature constraint in the deep moonquake source region. One is the Model 1 of Kuskov and Kronrod [1998]. Kuskov and Kronrod [1998] used thermodynamic and geochemical models with a seismic velocity model to construct the temperature profile. They used two different boundary conditions to construct two end-members for the temperature profiles, and Model 1 corresponds to the case where they assumed a lower limit of the density at the crust mantle boundary. Another model compatible with the brittle-ductile 
transition temperature is the wet model with $>0.1 \mathrm{wt} \%$ of water from Karato [2013]. As it was discussed before, under wet conditions, olivine strength will be lower by a factor of 1.3-1.6 [Girard et al., 2013] and its impact on the brittle-ductile transition temperature in the deep moonquake source regions is small.

To conclude, the cold models proposed in previous studies are compatible with our estimation of the brittleductile transition. For these models, deep moonquakes can be understood as the brittle response of the Moon due to tidal stress accumulation.

\section{Summary and Conclusion}

In this study, we reevaluated source parameters of deep moonquakes through spectral analyses. To improve our estimation, we combined both LP and SP seismic records to obtain continuous spectra and carried out spectral analysis with a broader frequency band. This enabled us to study more than 100 deep moonquake events from three different source regions and to perform a statistical and comparative investigation of the source parameters.

We showed that a traditional self-similar fault model gives stress drops of $\sim 0.1 \mathrm{MPa}$. Furthermore, our results support the self-affine fault model for deep moonquakes, also observed for terrestrial faults. Our results show that the roughness parameter (Hurst exponent) of the fault is significantly smaller compared to terrestrial faults, which implies that the deep moonquake faults are much smoother than the terrestrial counterparts. This results in stress drop of $0.05 \mathrm{MPa}$, which is comparable to shear tidal stress acting on deep moonquake faults. This suggests that the tidal stress not only triggers the deep moonquake activity but also acts as a dominant source of the excitation.

Assuming that the tidal stress is responsible for deep moonquake activity, we reevaluated the brittle-ductile transition temperature of deep moonquake source regions with tidal strain rate. This resulted in higher brittle-ductile transition temperature than the temperature previously proposed. Obtained transition temperatures were compared with temperature profiles proposed in previous studies. While most of the modeled temperature profiles are hotter than the transition temperature, cold temperature profiles model, such as the model with wet mantle from Karato [2013], are compatible with the brittle-ductile transition temperature obtained in this study.

\section{Appendix A: Computation of the Strain Rate}

We computed the strain rate at a given deep moonquake source with a simple model of the Earth tide, assuming a tidal potential of harmonic order 2 expressed as for Weber et al. [2009]:

$$
\phi_{2}=\frac{G M_{E} r^{2}}{2 r_{s}^{3}}\left(1-3 \cos ^{2} \Delta\right)
$$

where $G, M_{E}, r, r_{s}$, and $\Delta$ are the gravitational constant, Earth's mass, lunar radius, Earth to Moon distance, and angular distance between the Earth's nadir point on the Moon and the deep moonquake's epicenter. We first compute the vertical $U(r)$ and horizontal tidal displacement $V(r)$ from Wahr et al. [2009] software (https://code. google.com/p/satstress/) and then strain in spherical coordinate (see Phinney and Burridge [1973] for the strain and stress expressions). This consists in the resolution of the tide equation for a gravito-elastic lunar model, which can be rewritten as

$$
-\rho \omega^{2} \vec{u}=\vec{\nabla} \vec{T}-\vec{g} \vec{\nabla}(\rho \vec{u})-\rho \vec{\nabla} \phi_{1}-\rho \vec{\nabla} \phi_{2} \nabla^{2} \phi_{1}=-4 \pi G \vec{\nabla}(\rho \vec{u})
$$

where $\boldsymbol{T}$ is the stress tensor perturbation (depending linearly on $\mathbf{u}$ and which includes elastic stress and displacement in the prestressed body), $\phi_{1}$ is the mass redistribution potential, $\rho$ is the density, $g$ is the gravity, and $\omega$ is the tidal bulge angular frequency (in the lunar reference frame). See Lognonné and Clévédé for more details on the equation as well as associated boundary conditions.

We then decompose the angular term of equation (A1) in P20 and P22 harmonics, by using $\cos \Delta=\sin \theta \cos \phi$, such that

$$
P_{2}^{0}(\cos \theta)=-\frac{1}{2} P_{2}^{0}(\cos \theta)+\sqrt{\frac{3}{2}} P_{2}^{2}(\cos \theta) \cos (2 \phi)
$$

where $\theta$ and $\varphi$ are the colatitude and longitude with respect to a spherical coordinate system whose vertical axis is perpendicular to the Earth's nadir point direction. We then compute the time evolution of $\theta$ and $\varphi$ by 
Acknowledgments

We would like to acknowledge the reviewers, especially A. Khan for the comments and discussion which was important to improve the manuscript. This work has been supported by CNES and by ANR SISMOMARS. Taichi Kawamura would like to acknowledge the ANR SISMOMARS for the additional support for his Postdoc position. This is IPGP contribution 3865. The raw Apollo seismic data used in this study are available either through NASA Space Science Data Coordinate Archive (http:// nssdc.gsfc.nasa.gov/; PSPG-00665, PSPG-00012, and PSPG-00738) or IPGP Data Center (http://datacenter.jpgp.fr/ metadata/XA/) assuming a simple lunar orbit with semimajor great axis of $384,748 \mathrm{~km}$, eccentricity of 0.059006 , lunar radius of $1738 \mathrm{~km}$, and lunar orbit period of $T=27.555$ day, assuming that the $r_{s}$ distance verifies Kepler law, Moon is in synchronous rotation, and a lunar rotation axis tilted by $6.68^{\circ}$ with respect to the orbital plane and along the zero lunar meridian direction at the origin of time. The strain rate is computed numerically from the numerical expression with the time depending on radius, latitude, and longitude by time finite differences, and the maximum strain is then considered. For the Weber et al. [2009], the obtained strain is about $6.6 \times 10^{-13} \mathrm{~s}=5.7 \times 10^{-8} \mathrm{day}^{-1}$ for the horizontal shear strain, which leads, for the $\mu=10^{10} \mathrm{~Pa}=10^{5}$ bar rigidity, to stress of $2 \mu$ times the shear strain, and therefore of about $0.011 \mathrm{bar} / \mathrm{d}=1.1 \mathrm{kPa} / \mathrm{d}$, to be compared to Figure $9 \mathrm{~b}$ of Weber et al. [2009], which suggests peak-to-peak stress of $2 \times 0.015 \mathrm{bar} / \mathrm{d}$.

\section{References}

Aki, K., and P. G. Richards (2002), Quantitative Seismology, 2nd ed., chap. 10, pp. 491-536, Univ. Sci. Books, Sausalito, Calif.

Allmann, B. B., and P. M. Shearer (2009), Global variations of stress drop for moderate to large earthquakes, J. Geophys. Res., 114, B01310, doi:10.1029/2009JB005821.

Abercrombie, R. E. (2014), Stress drops of repeating earthquakes on the San Andreas Fault at Parkfield, Geophys. Res. Lett., 41, 8784-8791, doi:10.1002/2014GL062079.

Baker, S. F. (1999), Processing near-surface seismic reflection data: A primer (Society of Exploration Geophysicists Course Notes Volume 9), Soc. Explor. Geophys., doi:10.1190/1.9781560802020.

Bistacchi, A., W. A. Griffith, S. A. F. Smith, G. Toro, R. Jones, and S. Nielsen (2011), Fault roughness at seismogenic depths from lidar and photogrammetric analysis, Pure Appl. Geophys., doi:10.1007/s00024-011-0301-7.

Boettcher, M. S., G. Hirth, and B. Evans (2007), Olivine friction at the base of oceanic seismogenic zones, J. Geophys. Res., 112, B01205, doi:10.1029/2006JB004301.

Brodsky, E. E., J. J. Gilchrist, A. Sagy, and C. Collettini (2011), Faults smooth gradually as a function of slip, Earth Planet. Sci. Lett., 302, 185-193, doi:10.1016/j.epsl.2010.12.010.

Brodsky, E. E., J. D. Kirkpatrick, and T. Candela (2015), Constraints from fault roughness on the scale-dependent strength of rocks, Geology, doi:10.1130/G37206.1.

Candela, T., F. Renard, M. Bouchon, J. Schmittbuhl, and E. E. Brodsky (2011), Stress drop during earthquakes: Effect of fault roughness scaling, Bull. Seismol. Soc. Am., 101(5), 2369-2387.

Candela, T., F. Renard, Y. Klinger, K. Mair, J. Schmittbuhl, and E. E. Brodsky (2012), Roughness of fault surfaces over nine decades of length scales, J. Geophys. Res., 117, B08409, doi:10.1029/2011JB009041.

Chen, Y., Y. Zhang, Y. Liu, Y. Guan, J. Eiler, and E. M. Stolper (2015), Water, fluorine, and sulfur concentrations in the lunar mantle, Earth Planet. Sci. Lett., 427, 37-46.

Cheng, C. H., and M. N. Toksöz (1978), Tidal stresses in the Moon, J. Geophys. Res., 83, 845-853, doi:10.1029/JB083iB02p00845.

Collettini, C., A. Niemeijer, C. Viti, and C. Marone (2009), Fault zone fabric and fault weakness, Nature, 462, 907-910, doi:10.1038/nature08585

Dainty, A. M., M. N. Toksöz, K. R. Anderson, P. J. Pines, Y. Nakamura, and G. Latham (1974), Seismic scattering and shallow structure of the Moon in oceanus procellarum, Moon, 9, 11, doi:10.1007/BF00565388.

De Paola, N., T. Hirose, T. Mitchell, G. Di Toro, C. Viti, and T. Shimamoto (2011), Fault lubrication and earthquake propagation in thermally unstable rocks, Geology, 39(1), p35-p38, doi:10.1130/G31398.1.

Di Toro, G., R. Han, T. Hirose, N. De Paola, S. Nielsen, K. Mizoguchi, F. Ferri, M. Cocco, and T. Shimamoto (2011), Fault lubrication during earthquakes, Nature, 471, 494-498, doi:10.1038/nature09838.

Efron, B. (1979), Bootstrap methods: Another look at the jackknife, Ann. Stat., 7(1), 1-26, doi:10.1214/aos/1176344552. [Available at http:// projecteuclid.org/euclid.aos/1176344552.]

Elkins-Tanton, L. T., and T. L. Grove (2011), Water (hydrogen) in the lunar mantle: Results from petrology and magma ocean modeling, Earth Planet. Sci. Lett., 307, 173-179.

Frohlich, C., and Y. Nakamura (2009), The physical mechanisms of deep moonquakes and intermediate-depth earthquakes: How similar and how different?, Phys. Earth Planet. Inter., 173, 365-374.

Gagnepain-Beyneix, J., P. Lognonné, H. Chenet, D. Lombardi, and T. Spohn (2006), A seismic model of the lunar mantle and constraints on temperature and mineralogy, Phys. Earth Planet. Inter., 159, 140-166.

Garcia, R. F., J. Gagnepain-Beyneix, S. Chevrot, and P. Lognonné (2011), Very preliminary reference Moon model, Phys. Earth Planet. Inter., 188, 96-113.

Girard, J., J. Chen, P. Raterron, and C. W. Holyoke III (2013), Hydrolytic weakening of olivine at mantle pressure: Evidence of [100](010) slip system softening from single-crystal deformation experiments, Phys. Earth Planet. Inter., 216, 12-20.

Goins, N. R., A. M. Danity, and M. N. Toksöz (1981), Seismic energy release of the Moon, J. Geophys. Res., 86, 378-388, doi:10.1029/ JB086iB01p00378.

Harada, Y., S. Goossens, K. Matsumoto, J. Yan, J. Ping, H. Noda, and J. Haruyama (2014), Strong tidal heating in an ultralow-viscosity zone at the core-mantle boundary of the Moon, Nat. Geosci., 7, 569-572, doi:10.1038/ngeo2211.

Hacker, B. R., S. M. Peacock, G. A. Abers, and S. D. Holloway (2003), Subduction factory, 2, are intermediate-depth earthquakes in subducting slabs linked to metamorphic dehydration reactions?, J. Geophys. Res., 108(B1), 2030, doi:10.1029/2001JB001129.

Ide, S., G. C. Beroza, S. G. Prejean, and W. L. Ellsworth (2003), Apparent break in earthquake scaling due to path and site effects on deep borehole recordings, J. Geophys. Res., 108(B5), 2271, doi:10.1029/2001JB001617.

Karato, S. (2013), Geophysical constraints on the water content of the lunar mantle and its implications for the origin of the Moon, Earth Planet. Sci. Lett., 384, 144-153.

Khan, A., and K. J. Mosegaard (2002), An inquiry into the lunar interior: A non-linear inversion of the Apollo lunar seismic data, J. Geophys. Res., 107(E6), 5036, doi:10.1029/2001JE001658.

Khan, A., J. A. D. Connolly, J. Maclennan, and K. Mosegaard (2007), Joint Inversion of Seismic and Gravity Data for Lunar Composition and Thermal State, Geophys. J. Int., 168, 243, doi:10.1111/j.1365-246X.2006.03200.x.

Khan, A., J. A. D. Connolly, A. Pommier, and J. Noir (2014), Geophysical evidence for melt in the deep lunar interior and implications for lunar evolution, J. Geophys. Res. Planets, 119, 2197-2221, doi:10.1002/2014JE004661. 
Kuskov, O. L., and V. A. Kronrod (1998), Constitution of the Moon 5. Constraints on composition, density, temperature and radius of a core, Phys. Earth Planet. Inter., 107, 285-306.

Kuskov, O. L., and V. A. Kronrod (2009), Geochemical constraints on the model of the composition and thermal conditions of the Moon according to seismic data, Izv., Phys. Solid Earth, 45, 753, doi:10.1134/S1069351309090043.

Lammlein, D. R. (1977), Lunar seismicity and tectonics, Phys. Earth Planet. Inter., 14, 224-174.

Lognonné P., and C. Johnson (2007), Planetary seismology, in Treatise in Geophysics, Planets and Moons, vol. 10, chap. 4, edited by G. Shubert, pp. 69-122, Elsevier, Amsterdam, Netherlands, doi:10.1016/B978-044452748-6.00154-1.

Lognonné P., and C. Johnson (2015), Planetary seismology, in Treatise in Geophysics, Planets and Moons, vol. 10, 2nd ed., chap. 10.03, edited by G. Shubert, pp. 65-120, Elsevier, Amsterdam, Netherlands, doi:10.1016/B978-0-444-53802-4.00167-6.

Lognonné, P., J. Gagnepain-Beyneix, and H. Chenet (2003), A new seismic model of the Moon: Implication in terms of structure, formation and evolution, Earth Planet. Sci. Lett., 211, 27-44.

Mayeda, K., and R. W. Walter (1996), Moment, energy, stress drop, and source spectra of western United States earthquakes from regional coda envelopes, J. Geophys. Res., 101(B5), 11,195-11,208, doi:10.1029/96JB00112.

McKenzie, D., J. Jackson, and K. Priestley (2005), Thermal structure of oceanic and continental lithosphere, Earth Planet. Sci. Lett., 233, 337-349.

Minshull, T. A., and N. R. Goulty (1988), The influence of tidal stresses on deep moonquake activity, Phys. Earth Planet. Inter., 52, 41-55.

Nakamura, Y. (1992), Lunar-Catalog for lunar seismic data from Apollo passive seismic experiment on 8-mm video cassette (EXABYTE) tapes (University of Texas Institute for Geophysics).

Nakamura, Y. (1978), A1 moonquakes-Source distribution and mechanism, Lunar and Planet. Sci. Conf., 9th, Houston, Tex., 13-17 March. Nakamura, Y. (2003), New identification of deep moonquakes in the Apollo lunar seismic data, Phys. Earth Planet. Inter., 139, 197-205.

Nakamura, Y. (2009), Farside deep moonquakes and deep interior of the Moon, J. Geophys. Res., 110, E01001, doi:10.1029/2004JE002332.

Nakamura, Y., and J. Koyama (1982), Seismic Q of the lunar upper mantle, J. Geophys. Res., 87(B6), 4855-4861, doi:10.1029/JB087iB06p04855.

Ohuchi, T., T. Kawazoe, Y. Higo, K. Funakoshi, A. Suzuki, T. Kikegawa, and T. Irifune (2015), Dislocation-accommodated grain boundary sliding as the major deformation mechanism of olivine in the Earth's upper mantle, Sci. Adv., 1, e1500360.

Phinney, R. A., and R. Burridge (1973), Representation of the elastic-gravitational excitation of a spherical Earth model by generalized spherical harmonics, Geophys. J. R. asfr. SOC., 34, 451-487.

Reches, Z., and D. A. Lockner (2010), Fault weakening and earthquake instability by powder lubrication, Nature, 467, 452-455, doi:10.1038/ nature09348.

Saal, A. E., E. H. Hauri, M. Lo Cascio, J. A. Van Orman, M. J. Rutherford, and R. F. Cooper (2008), Volatile content of lunar volcanic glasses and the presence of water in the Moon's interior, Nature, 454, 193-195.

Schmittbuhl, J., S. Gentier, and S. Roux (1993), Field measurements of the roughness of fault surfaces, Geophys. Res. Lett., 20, 639-641, doi:10.1029/93GL00170.

Schmittbuhl, J., G. Chambon, A. Hansen, and M. Bouchon (2006), Are stress distributions along faults the signature of asperity squeeze? Geophys. Res. Lett., 33, L13307, doi:10.1029/2006GL025952.

Shearer, P. M. (2009), Introduction to Seismology, 2nd ed., chap. 9, pp. 241-300, Cambridge Univ. Press, U.K.

Toksöz, M. N., N. R. Goins, and C. H. Cheng (1977), Moonquakes: Mechanisms and relation to tidal stresses, Science, 196, 979-981.

Wahr, J., Z. A. Selvans, M. E. Mullen, A. C. Barr, G. Collins, M. M. Selvans, and R. T. Pappalardo (2009), Modeling stresses on satellites due to nonsynchronous rotation and orbital eccentricity using gravitational potential theory, Icarus, 200, 188-206.

Weber, R. C., B. G. Bills, and C. L. Johnson (2009), Constraints on deep moonquake focal mechanisms through analyses of tidal stress, J. Geophys. Res., 114, E05001, doi:10.1029/2008JE003286.

Weber, R. C., P. Lin, E. J. Garnero, Q. Williams, and P. Lognonné (2011), Seismic detection of the lunar core, Science, 331(6015), $309-312$.

Ye, L., T. Lay, H. Kanamori, Z. Zhan, and Z. Duputel (2016), Diverse rupture processes in the 2015 Peru deep earthquake doublet, Sci. Adv., 2(6), e1600581, doi:10.1126/sciadv.1600581. 